\title{
Anemia Due to Inflammation in an Anti-Coagulated Patient with Blue Rubber Bleb Nevus Syndrome
}

\author{
Aldo Bonaventura ${ }^{1}$, Luca Liberale ${ }^{1}$, Nadia Hussein El-Dib ${ }^{1}$, \\ Fabrizio Montecucco ${ }^{1,2}$, Franco Dallegri ${ }^{1}$ \\ ${ }^{1}$ First Clinic of Internal Medicine, Department of Internal Medicine, University of Genoa School of Medicine. \\ IRCCS Azienda Ospedaliera Universitaria San Martino-IST Istituto Nazionale per la Ricerca sul Cancro, 6 viale Benedetto XV, 16132 Genoa, Italy \\ ${ }^{2}$ Division of Cardiology, Foundation for Medical Researches, Department of Medical Specialties, University of Geneva, 64 Avenue de la Roseraie, \\ 1211 Geneva, Switzerland
}

\begin{abstract}
SUMMARY
Background: Blue rubber bleb nevus syndrome (BRBNS) is a rare disease characterized by vascular malformations mostly involving skin and gastrointestinal tract. This disease is often associated with sideropenic anemia and occult bleeding.

Methods: We report the case of chronic severe anemia in an old patient under oral anticoagulation treatment for chronic atrial fibrillation.

Results: At admission, the patient also presented fever and increased laboratory parameters of systemic inflammation (ferritin $308 \mathrm{mcg} / \mathrm{L}$, C-reactive protein (CRP) $244 \mathrm{mg} / \mathrm{L}$ ). A small bluish-colored lesion over the left ear lobe was observed. Fecal occult blood test was negative as well as other signs of active bleeding. Lower gastrointestinal endoscopy revealed internal hemorrhoids and multiple teleangiectasias that were treated with argon plasma coagulation. Videocapsule endoscopy demonstrated multiple bluish nodular lesions in the small intestine. Unexpectedly, chronic severe anemia due to systemic inflammation was diagnosed in an old anticoagulated patient with BRNBS. The patient was treated with blood transfusions, hydration, antibiotic treatment, and long-acting octreotide acetate, without stopping warfarin. Fever and inflammation disappeared without any acute gastrointestinal bleeding and improvement of hemoglobin levels at three-month follow up.

Conclusions: This is the oldest patient presenting with chronic anemia, in which BRNBS was also diagnosed. Surprisingly, anemia was mainly caused by systemic inflammation instead of chronic gastrointestinal bleeding. However, we would recommend investigating this disease also in old subjects with mild signs and symptoms.
\end{abstract}

(Clin. Lab. 2016;62:241-243. DOI: 10.7754/Clin.Lab.2015.150617)

Correspondence:

Fabrizio Montecucco, MD, PhD

Division of Laboratory Medicine

Department of Genetics and Laboratory Medicine

Geneva University Hospitals

4 rue Gabrielle-Perret-Gentil

1205 Geneva, Switzerland

Phone: +41223827238

Fax: $\quad+41223827245$

Email: fabrizio.montecucco@unige.ch

\section{KEY WORDS}

blue rubber bleb nevus syndrome, anemia, inflammation

\section{INTRODUCTION}

Blue rubber bleb nevus syndrome (BRBNS), also called Bean's syndrome, is a rare disease characterized by vascular malformations mostly involving skin and gastrointestinal tract. Occult bleeding and sideropenic anemia are common clinical presentation in BRBNS [1]. 


\section{CASE REPORT}

The case of chronic anemia due to persistent inflammation in an 83-year-old man with BRNBS and under oral anticoagulation treatment was reported. He suffered from chronic coronary artery disease (CAD), permanent atrial fibrillation under oral anti-coagulation treatment, chronic obstructive pulmonary disease (COPD), essential hypertension, and type 2 diabetes. In addition, the patient did a radical prostatectomy because of a localized cancer several years before. In the five months prior to admission to our Division, the patient was hospitalized in another hospital for severe dyspnea and sideropenic anemia (hemoglobin [Hb] $7.2 \mathrm{~g} / \mathrm{dL}$, mean corpuscular volume [MCV] $69.9 \mathrm{fL}$, ferritin $14 \mathrm{mcg} / \mathrm{L}$, positive occult fecal blood). The gastroscopy demonstrated a hyperemic gastritis. The colonscopy identified coecal angiodysplasias and multiple, small venous ecstasies in the descendent colon that was not treated due to anti-coagulation treatment. After intravenous iron supplementation, the patient was discharged with oral anti-coagulation treatment and oral iron supplementation (for three weeks). At admission in our Division, the patient presented fever and anemia. Laboratory findings revealed $\mathrm{Hb} 7.8 \mathrm{~g} / \mathrm{dL}$ with a $\mathrm{MCV}$ of $82.3 \mathrm{fL}$, reticulocyte count $27900 / \mathrm{mm}^{3}$ and ferritin $308 \mathrm{mcg} / \mathrm{L}$, INR 2.66, C-reactive protein (CRP) $244 \mathrm{mg} / \mathrm{L}$, creatinine $2.1 \mathrm{mg} / \mathrm{dL}$. Clinical examination showed mild, dry cough, a mild systolic murmur, a pale colour of the skin, and a small bluish-colored lesion over the left ear lobe. Chest X-ray showed right basal, pleural opacity due to loculated, pleural effusion or previous pleuritis; computed tomography (CT)-guided thoracentesis showed no malignant cells and negative real time polymerase chain reaction for M. tuberculosis. Despite internal hemorrhoids grade 1, the patient did not present any acute major bleeding. Brain CT scan excluded both brain hemorrhage and vascular malformations. Fecal occult blood test was negative. The patient underwent upper and lower gastrointestinal endoscopy, the first one negative for acute bleeding. Colonscopy revealed multiple colic venous ecstasias and coecal and right colic teleangiectasias, the latter treated with argon plasma coagulation. In addition, videocapsule endoscopy demonstrated multiple bluish nodular lesions in the small intestine suggestive for BRNBS (Figure 1). During the 21-day hospitalisation, the patient received three blood transfusions, maintaining a hemoglobin around $9.5 \mathrm{~g} / \mathrm{dL}$. Under appropriate hydration and antibiotic treatment fever disappeared and CRP and creatinine improved before discharge to $30.3 \mathrm{mg} / \mathrm{L}$ and $1.1 \mathrm{mg} / \mathrm{dL}$, respectively. Considering chronic atrial fibrillation, owing to $\mathrm{CHA}_{2} \mathrm{DS}_{2}-\mathrm{VASc}$ score of 5 and HAS-BLED score of 2, warfarin was not stopped maintaining INR between 2 and 2.5. We started treatment with monthly intramuscular injections of long-acting octreotide acetate and no gastrointestinal bleeding occurred since the 3-month follow-up. The last $\mathrm{Hb}$ value was $9.6 \mathrm{~g} / \mathrm{dL}$.

\section{DISCUSSION}

In this old patient with BRBNS, we diagnosed chronic severe anemia due to systemic inflammation during COPD exacerbation. We planned to monitor patient with monthly hemochrome, while endoscopy will be considered only in case of acute bleeding or rapid anemization. The challenge was to decide on continuing with anti-coagulation because of atrial fibrillation. Once treated right colic teleangiectasias and excluded acute bleeding of bluish lesions in small intestine, we decided to carry on with warfarin maintaining acceptable levels of $\mathrm{Hb}$.

This patient was the oldest subject ( 83 years old) reported in literature [2] that presented with BRBNS and concomitant chronic anemia. BRNBS develops sporadically, but there are forms inherited with an autosomal dominant pattern involving chromosome 9p [3]. Patients have a normal life span and no tendency to malignancy is described in literature. The extent of visceral organ involvement is determinant for the prognosis. Typically, skin lesions may present at birth or in the neonatal period, but sometimes even later in life; their sizes vary from a few millimeters to some centimeters and localize in the trunk and upper extremities. Three different types of lesions are described; the most common one is red to bluish, rubbery, easily compressible, and slowly refillable on release of pressure, this is to be considered as an important diagnostic sign. Bluish nevi can also be present in the duodenum and colon during gastrointestinal endoscopy [4]. Involvement of other organs and tissues is considered rare resulting in pulmonary hypertension, hemopericardium and hemothorax, symptoms of the central nervous system, lesions of glans penis, vulva, and eyes; orthopedic abnormalities are often present [5]. Our patient did not present any of these additional symptoms and signs. Although several treatments are available (anti-angiogenetic agents such as steroids, propranolol, octreotide, and interferon $\alpha-2 a$ ) [6-8], we decided to use octreotide after laser photocoagulation without any guarantee of durable effects. We excluded a surgical approach, considered aggressive and unhelpful because lesions could recur after removal [9]. Considering this case, in which the major cause of anemia was represented by systemic inflammation during COPD exacerbation, we would recommend also checking other causes (such as BRNBS) in old subjects with mild signs and symptoms of the disease.

\section{Declaration of Interest:}

None declared. 

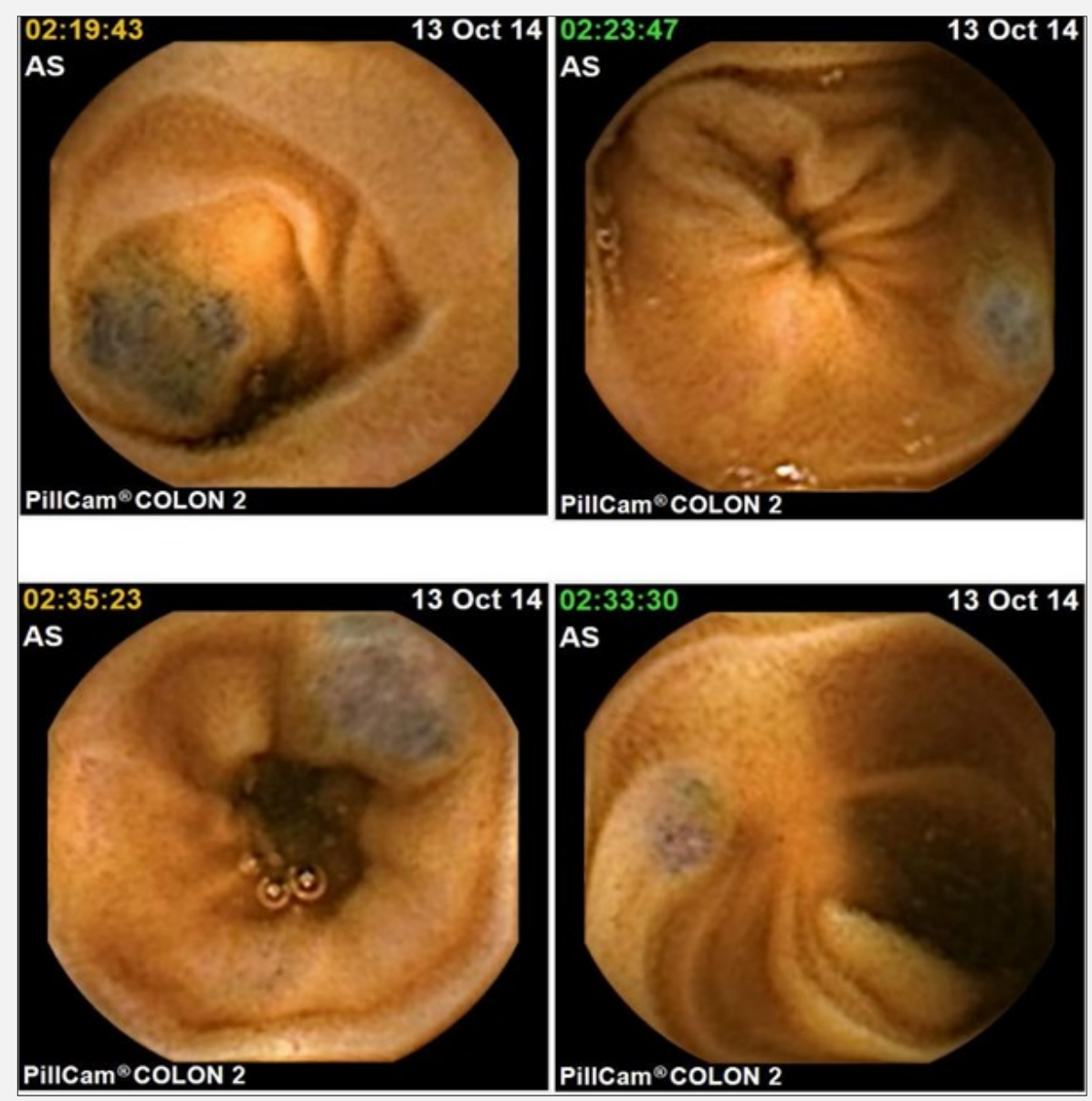

Figure 1. Multiple bluish nodular lesions in different tracts of the small intestine.

\section{References:}

1. Bean WB. Blue rubber bleb nevus of skin and gastro-intestinal tract. in: W.B. Bean (Ed.) Vascular Spiders and Related Lesions of the Skin. Charles Thomas Publishers, Springfield (Ill);958: $178-85$.

2. Tomelleri G, Cappellari M, Di Matteo A, et al. Blue rubber bleb nevus syndrome with late onset of central nervous system symptomatic involvement. Neurol Sci 2010;31:501-4.

3. Gallione CJ, Pasyk KA, Boon LM, et al. A gene for familial venous malformations maps to chromosome $9 \mathrm{p}$ in a second large kindred. J Med Genet 1995;32:197-9.

4. Oksüzoğlu BC, Oksüzoğlu G, Cakir U, Bayir T, Esen M. Blue rubber bleb nevus syndrome. Am J Gastroenterol 1996;91:780782 .

5. McCarthy JC, Goldberg MJ, Zimbler S. Orthopaedic dysfunction in the blue rubber-bleb nevus syndrome. J Bone Joint Surg Am 1982;64: $280-3$
6. Hansen LF, Wewer V, Pedersen SA, Matzen P, Paerregaard A Severe blue rubber bleb nevus syndrome in a neonate. Eur J Pediatr Surg 2009;19: 47-9.

7. Gonzalez D, Elizondo BJ, Haslag S, et al. Chronic subcutaneous octreotide decreases gastrointestinal blood loss in blue rubberbleb nevus syndrome. J Pediatr Gastroenterol Nutr 2001;33:1838 .

8. Buckmiller LM, Munson PD, Dyamenahalli U, Dai Y, Richter GT. Propranolol for infantile hemangiomas: early experience at a tertiary vascular anomalies center. Laryngoscope 2010;120:67681.

9. Fishman SJ, Smithers CJ, Folkman J, et al. Blue rubber bleb nevus syndrome: surgical eradication of gastrointestinal bleeding. Ann Surg 2005;241:523-8. 